\title{
Economía popular solidaria y su efecto en el desarrollo económico comunal
}

\section{Popular solidarity economy and its effect on community economic development}

\author{
Diana Asunción Bravo Vélez \\ dabravo@utm.edu.ec \\ Universidad Técnica de Manabí \\ Ecuador \\ https://orcid.org/0000-0002-5323-1625 \\ Carlos Artemidoro Zea Barahona \\ carlos.zea@unesum.edu.ec \\ Universidad Estatal del Sur de Manabí \\ Ecuador \\ https://orcid.org/0000-0001-7546-7148 \\ José Alfredo FrancoYoza \\ jose.franco@unesum.edu.ec \\ Universidad Estatal del Sur de Manabí \\ Ecuador \\ https://orcid.org/0000-0001-6204-9692 \\ Tatiana Lisbeth Mero Flores \\ tatiana.mero@unesum.edu.ec \\ Universidad Estatal del Sur de Manabí \\ Ecuador \\ https://orcid.org/0000-0003-4952-6292
}

Recibido: 18 de mayo del 2019

Aprobado: 16 de junio del 2019

\section{RESUMEN}

La presente investigación tuvo por objetivo analizar la Economía popular y su efecto en el desarrollo económico de la comuna Agua Blanca. Se enfocó desde la descriptiva estadística, se construyó un baremo de categoría, lo cual indicó que la población de estudio se encuentra en el rango muy deficiente efecto en el desarrollo 
económico de la comuna Agua Blanca. Lo cual refleja que existe escaso o nulo conocimiento y aplicación políticas relacionadas a la economía popular y solidaria, lo cual implica formarse para establecer acciones que le permitan empoderarse de estrategias fortalecedoras de un accionar en favor de tener en cuenta de forma tangible un desarrollo económico sostenible en aras de promover cambios significativos y duraderos para la comunidad.

Descriptores: Economía colectiva; Sistema económico; Economía mixta; Alianza pública privada.

\begin{abstract}
The purpose of this research was to analyze the Popular Economy and its effect on the economic development of the Agua Blanca commune. It was focused from the statistical description, a category scale was constructed, which indicated that the study population is in the very poor range effect on the economic development of the Agua Blanca commune. This reflects that there is little or no knowledge and application of policies related to the popular and solidarity economy, which implies training to establish actions that allow it to empower itself with strategies that strengthen an action in favor of tangibly taking into account sustainable economic development in order to promote significant and lasting changes for the community.
\end{abstract}

Descriptors: Collective economy; Economic systems, Mixed economy, Public private partnerships.

\title{
INTRODUCCIÓN
}

La ley de la economía popular y solidaria propende a una relación dinámica, equilibrada, entre sociedad, Estado y mercado. Es decir que está relacionada directamente con las diferentes asociaciones, entidades o agrupaciones de un determinando sector con la finalidad de que puedan desarrollarse socioeconómicamente mediante apoyos financieros productivos, transformándolos en la creación de una microempresa que oferte un bien o servicio y poder mejorar los ingresos de las familias y por ende la calidad de vida.

El desarrollo económico de un determinado país se fundamenta en generar patrimonio o riquezas mediante la asignación de recursos con el fin de mantener una estabilidad económica y el bienestar de los habitantes, evidentemente la comuna 
Agua Blanca desarrolla económicamente gracias a todos los atractivos turísticos que posee, lugar que es visitado consecutivamente por turistas nacionales y extranjeros. En Ecuador existe la ley de la Economía Popular y Solidaria aprobada con la finalidad de establecer un sistema económico, social, solidario, incluyendo a los sectores cooperativistas, asociativos, comunitarios, con el propósito de beneficiar a las personas más vulnerables implantando proyectos y creando microempresas para tener mejoramiento económico. La comuna Agua Blanca es una comunidad que en la actualidad no puede desarrollarse sin la actividad turística, su progreso está encaminado a todos los atractivos que oferta al turista, entre los cuales están senderismo, alojamiento, servicios de alimentación, museos, miradores y los productos medicinales que poseen las aguas de la laguna azufrada.

La ley de la economía popular y solidaria fortalece el desarrollo socioeconómico del Ecuador, como se lo menciona en el Art. 1 (Ley Orgánica de la Economia Popular y solidaria, 2011).

Se la define como una forma de organización economía, donde sus integrantes, individual o colectivamente, organizan y desarrollan procesos de producción, intercambio, comercialización, financiamiento y consumo de bienes y servicios, para satisfacer necesidades y generar ingresos, basadas en relaciones de solidaridad, cooperación y reciprocidad, privilegiando al trabajo y al ser humano como sujeto y fin de su actividad, orientada al buen vivir, en armonía con la naturaleza, por sobre la apropiación, el lucro y la acumulación de capital (p. 3)

La comuna Agua Blanca es una zona donde se realiza el turismo comunitario, es un sitio que posee riquezas naturales atrae constantemente a turistas propios y extraños, se desconoce si la economía popular y solidaria incide en el desarrollo económico del antes mencionado lugar. Las organizaciones comunitarias de la comuna Agua Blanca aportan significativamente al desarrollo económico, por cuanto generan un espacio de emprendimientos, intercambio y comercialización de bienes, productos, servicios patrimoniales de la comuna de este cantón costero, consiguiendo de esta manera resaltar el gran potencial turístico que posee por cuanto cuenta con una riqueza paisajística, histórica y cultural. Es así que la actual 
investigación tiene por objetivo analizar la Economía popular y su efecto en el desarrollo económico de la comuna Agua Blanca.

\section{REFERENCIAL TEÓRICO}

\section{La economía popular y solidaria en el Ecuador}

La economía popular y solidaria en un principio constitucional tal como lo señalan Quevedo Vázquez, Vásquez Labefre \& Quevedo Barros (2019), quienes indican que esta tipología coloca al ser humano como centro del proceso productivo, otorgándole la posibilidad de crecer integralmente en la medida que es apoyado para el establecimiento de una gestión económica con base en diversos enfoques para la producción de bienes y servicios. La superintendencia de economía popular y solidaria (2019), plantea que:

La economía popular y solidaria es la forma de organización económica, donde sus integrantes, individual o colectivamente, organizan y desarrollan procesos de producción, intercambio, comercialización, financiamiento y consumo de bienes y servicios, para satisfacer necesidades y generar ingresos (p. 1).

Este modelo permite a las comunas organizarse para desarrollar acciones económicas que les favorezcan para su crecimiento, desde una óptica en pro de construir país para el buen vivir, siendo necesario tener una visión deontológica que compagine el accionar humanista desde una cosmovisión basada en el crecer cooperativo de los pueblos, focalizándose así, la posibilidad de contar con un desarrollo económico sostenible y sustentable.

Desde lo planteado, el desarrollo económico tiene un gran campo de aplicación práctica: se trata de la formulación, crítica, fundamentación y aplicación de la política económica del desarrollo. En ello participan de manera directa o indirecta, con planteamientos diversos y contradictorios, gobiernos nacionales y locales, organismos multinacionales, corporaciones privadas, organismos no 
gubernamentales, partidos y asociaciones políticas, iglesias, etcétera. (Zermeño, 2004).

La medición del desarrollo económico se ha realizado recurriendo a múltiples criterios que van desde la determinación de rangos de ingresos per cápita, el grado de industrialización alcanzado, la persistencia de estructuras productivas duales y las disparidades en la distribución del ingreso, hasta los cambios institucionales que modifican las reglas y las formas cómo interactúan los agentes económicos dentro de una sociedad. (Ocegueda, 2000)

En la actualidad, el desarrollo económico está íntimamente ligado al análisis coyuntural; para realizar este seguimiento se puede optar por la realización de un modelo econométrico regional, o bien el seguimiento de la coyuntura económica a través de indicadores sintéticos de actividad. Se denomina indicador sintético a cualquier combinación de indicadores individuales, a los cuales nos referimos como indicadores componentes o parciales. (Mondéjar J., Vargas V., \& Meseguer S., 2009).

En la perspectiva del desarrollo económico de un país no hay duda que una población mayoritariamente rural y agrícola que vive en condiciones precarias y el analfabetismo constituyen obstáculos para el desarrollo, pero también es cierto que el incremento de la población urbana no garantiza el desarrollo por la insuficiencia de la demanda laboral en las actividades industriales y la deficiente capacitación de la población que migra a las ciudades. Ciertamente que este es un problema complejo difícil de ser abordado en toda su dimensión (...) (CEPAL, 2013)

El problema del desarrollo económico del Ecuador se plantea en términos relativamente simples; los recursos en tierras aprovechables y en brazos están mal distribuidos; los niveles de consumo popular son bajos y dejan escaso margen para el ahorro; la población crece a ritmo acelerado. En cambio, es poco lo que se extrae del suelo en comparación con lo que se lograría mediante un aprovechamiento adecuado y extensivo. (Ibídem)

En Manabí, al hablar de desarrollo económico, se evidencia en las principales actividades a la que se dedican los manabitas, la primera es la agricultura. La provincia no solo destaca por la extensión territorial de 
Diana Asunción Bravo Vélez; Carlos Artemidoro Zea Barahona; Jose Alfredo Blanco Yoza; Tatiana Lisbeth Mero Flores

18.939,6 km2, sino también por su estabilidad climática. Cuenta con 1'583.000 hectáreas de tierra utilizada, que equivale al $84 \%$ de la región y el $13 \%$ del total del país. (EITelegrafo, 2016)

La pesca ocupa el segundo lugar y tiene un gran valor para los habitantes. Aunque no hay datos exactos del número de personas que trabajan en este sector, solo en la industria atunera laboran cerca de 12.000 personas. Eso incluye el proceso de industrialización del atún y la sardina. (Ibídem). En la localidad hay más de 300 barcos industriales con capacidad para almacenar 2.000 toneladas métricas. También cuentan con un promedio de 3.000 embarcaciones artesanales y con los barcos camaroneros constituyen la mayor flota pesquera del país. Esto convierte a Manta en el primer puerto pesquero de Ecuador y el primero en desembarque de atún a lo largo del Pacífico Sur Oriental. (Ibídem).

\section{METODOLOGÍA DE LA INVESTIGACIÓN}

La investigación se apoyó en lo descriptivo, (Hernández Sampieri, Fernández Collado \& Baptista 2014), lo cual permitió construir los resultados a partir de la información recopilada, la población estuvo conformada por treinta cinco personas con la finalidad de conocer la economía popular y su efecto en el desarrollo económico de la comuna Agua Blanca, describiéndose los hechos tal como ocurren en la realidad, evitándose la manipulación deliberada por parte de los investigadores, aplicándose un instrumento tipo escala de Likert de 24 ítems con cuatro alternativas de respuestas, cada una de ellas se les asignó un valor numérico siendo estas: siempre, (5), casi siempre (4), algunas veces (3), casi nunca (4), nunca (1), el cual obtuvo un Alfa de Cronbach de 0,790 , catalogándose como confiable.

Para el análisis de los datos estadísticos se procedió a construir un baremo de medición estadístico por medio del cual se calculó la media aritmética para realizar una comparación entre los rangos del baremo de medición y los datos obtenidos, lo que permitió establecer cuál era el nivel o categoría en el cual se encontraba la muestra de estudio. El cálculo de la media aritmética como medida de tendencia central por indicadores, dimensiones, variable de estudio; siendo así; que el 


\section{CIENCIAMATRIA}

Revista Interdisciplinaria de Humanidades, Educación, Ciencia y Tecnología

Año VI. Vol. VI. №10. Enero - Julio 2020

Hecho el depósito de ley: pp201602FA4721

ISSN-L: 2542-3029; ISSN: 2610-802X

Universidad Nacional Experimental Francisco de Miranda (UNEFM). Santa Ana de Coro. Venezuela

Diana Asunción Bravo Vélez; Carlos Artemidoro Zea Barahona; Jose Alfredo Blanco Yoza; Tatiana Lisbeth Mero Flores

resultado de este análisis permitió, "evaluar" el desempeño de cada indicador con respecto a la dimensión y a su vez con la variable de estudio, esto con la finalidad de seguir lo establecido por Kerlinger \& Lee (2008).

RESU

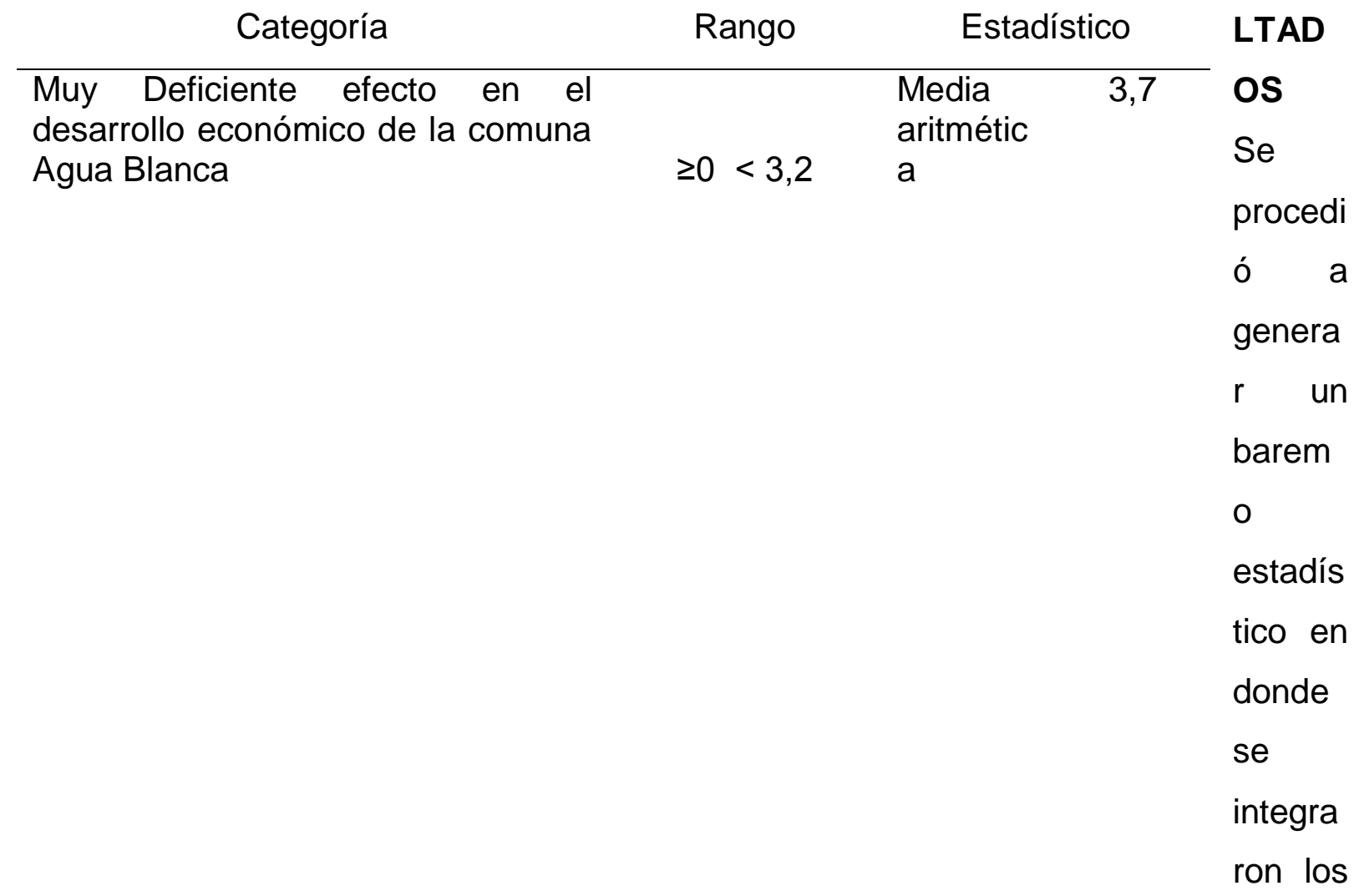

resultados significativos de las variables de estudio, esto permitió considerar el rango en el cual se encuentran en función de lo planteado en el objetivo de investigación.

Economía popular y su efecto en el desarrollo económico de la comuna Agua Blanca 
Deficiente Muy Deficiente efecto en el desarrollo económico de la Cuadr comuna Agua Blanca

$$
\geq 3,2<6,4
$$

Regular efecto en el desarrollo económico de la comuna Agua Blanca

Buen efecto en el desarrollo económico de la comuna Agua Blanca

Excelente efecto en el desarrollo $\quad \geq 14,8<18$ económico de la comuna Agua Blanca

En concordancia con los datos recopilados se puede evidenciar que las personas objeto de estudio, obtuvieron una media aritmética de 3,7; ubicándose en la categoría "Muy deficiente efecto en el desarrollo económico de la comuna Agua Blanca. Partiendo de lo expuesto, se tiene que los sujetos investigados consideran que no se ha producido un efecto significativo e importante por parte de las políticas planteadas por la economía popular y solidaria en la consolidación de un desarrollo económico acorde para el buen vivir desde el marco de promover acciones favorables para el sostenimiento y sustentabilidad de la sociedad. 


\section{CONCLUSIONES}

La comuna Agua Blanca al encontrarse un rango muy deficiente, refleja que existe escaso o nulo conocimiento y aplicación políticas relacionadas a la economía popular y solidaria, lo cual implica formarse para establecer acciones que le permitan empoderarse de estrategias fortalecedoras de un accionar en favor de tener en cuenta de forma tangible un desarrollo económico sostenible en aras de promover cambios significativos y duraderos para la comunidad, así se encontraría en concordancia con lo planteado por Aldana Zavala \& Colina Ysea (2019), quienes exponen la necesidad de fomentar una economía sostenible donde las personas involucradas se puedan fortalecer en la medida que tienen la oportunidad de emprender para el crecimiento integral de los mismos, visualizando la economía como un factor esencial para la evolución del ser humano, siempre y cuando esta se acompañe de una formación ética pensada para la vida en equilibrio con el medio donde se desenvuelve la persona.

\section{REFERENCIAS CONSULTADAS}

1. Aldana Zavala, J., \& Colina Ysea, F. (2019). Marketing verde en la conformación de una ciudadanía planetaria en el ámbito educativo latinoamericano. Revista San Gregorio, O(31), 150-161. Recuperado de http://revista.sangregorio.edu.ec/index.php/REVISTASANGREGORIO/article/v iew/972/15

2. Boza-Valle, J. A., \& Manjarez-Fuentes, N. (Agosto de 2016). Diagnóstico estratégico de emprendimientos de economía popular y solidaria en ecuador. Scielo, XXXVII(2). Obtenido de http://scielo.sld.cu/scielo.php?pid=S1815$\underline{59362016000200010 \& s c r i p t=s c i}$ arttext\&tlng=en

3. CEPAL. (2013). El desaerrollo económico del Ecuador. CEPAL, Secretaría de la Comisión Econóica para América Ltina y el Caribe. México: Editogran S.A. Obtenido de http://www.politicaeconomica.gob.ec/wpcontent/uploads/downloads/2014/02/librocepal.pdf

4. Coraggio, J. L. (1998). Economía urbana: la perspectiva popular (Segunda ed.). (r. illustrated, Ed.) Quito, Pichincha, Ecuador: Editorial Abya Yala. 
Diana Asunción Bravo Vélez; Carlos Artemidoro Zea Barahona; Jose Alfredo Blanco Yoza; Tatiana Lisbeth Mero Flores

Recuperado el 20 de Abril de 2016, de https://books.google.com.ec/books?id=UkE4pQZKColC\&printsec=frontcover\& $\mathrm{dq}=$ economia + popular $+\mathrm{y}+$ solidaria\&hl=es419\&sa=X\&ved=0ahUKEwiy0M6U25PLAhVBmh4KHVeHBtY4ChDoAQhJMAk $\# \mathrm{v}=$ onepage \& $\mathrm{q} \& \mathrm{f}=\mathrm{false}$

5. Coraggio, J. L. (Junio de 2014). La presencia de la economía social y solidaria y su institucionalización en América Latina. ECONSTOR(7), 22. Obtenido de https://www.econstor.eu/bitstream/10419/148805/1/862974798.pdf

6. El Telegrafo. (23 de Abril de 2016). La riqueza económica de Manabí se centra en la agricultura, la pesca y el turismo. Redacción Actualidad. Obtenido de http://www.eltelegrafo.com.ec/noticias/ecuador/3/la-riqueza-economica-demanabi-se-centra-en-la-agricultura-la-pesca-y-el-turismo

7. Guerra, P. (2012). La economía solidaria en Latinoamérica. PAPELES, 76. Obtenido http://www.fuhem.es/revistapapeles/docprivados/110/la\%20economia\%20soli daria\%20en\%20Latinoamerica P.\%20GUERRA.pdf

8. Hernández Sampieri, R., Fernández Collado , C., \& Baptista , L. (2014). Metodología de la investigación. México: Editorial Mc - Graw - Hill Interamericana. Sexta edición.

9. Kerlinger, F. \& Lee, H. (2008). Investigación del comportamiento: Métodos de investi-gación en Ciencias Sociales (4a . ed.). México: Mc Graw Hill.

10.Ley Orgánica de la Economia Popular y solidaria. (14 de abril de 2011). 48. Quito Pichincha, Ecuador. Obtenido de http://www.seps.gob.ec/documents/20181/25522/Ley\%20Orga\%CC\%81nica \%20de\%20Economi\%CC\%81a\%20Popular\%20y\%20Solidaria.pdf/0836bc47bf63-4aa0-b945-b94479a84ca1

11. Ley Orgánica de la Economia Popular y Solidaria. (2011). Obtenido de http://www.seps.gob.ec/documents/20181/25522/Ley\%20Orga\%CC\%81nica \%20de\%20Economi\%CC\%81a\%20Popular\%20y\%20Solidaria.pdf/0836bc47bf63-4aa0-b945-b94479a84ca1

12.Mondéjar J., J., Vargas V., M., \& Meseguer S., M. L. (2009). Medición del desarrollo económico regional a través de indicadores sintéticos de actividad. Revista Venezolana de Análisis de Coyuntura, XIV(2). Obtenido de http://www.redalyc.org/articulo.oa?id=36414215

13. Ocegueda, J. M. (2000). Crecimiento y desarrollo económico--el estado actual del debate. Baja California, México: UABC. Obtenido de 
https://books.google.com.ec/books?id=y5fGeXERg1UC\&printsec=frontcover\& $\mathrm{dq}=$ desarrollo+econ\%C3\%B3mico\&hl=es\&sa=X\&ved=0ahUKEwiks4ngqvbYA hUH1IMKHatHDulQ6AEIJTAA\#v=onepage\&q=desarrollo\%20econ\%C3\%B3mi co\&f=false

14. Quevedo Vázquez, J., Vásquez Labefre, L., \& Quevedo Barros, M. (2019). Análisis de las incubadoras de empresas en el Ecuador. Revista Arbitrada Interdisciplinaria Koinonía, 4(8), 633-648. doi:http://dx.doi.org/10.35381/r.k.v4i8.318

15.Superintendencia de economía popular y solidaria (2019). Conoce que es la Economía Popular y Solidaria (EPS). Recuperado de https://www.seps.gob.ec/noticia?conoce-la-eps

16.Zermeño, F. (2004). Lecciones de desarrollo económico. Plaza y Valdes. Obtenido de https://books.google.com.ec/books?id=Iv24eXOtNZ8C\&printsec=frontcover\&d $\mathrm{q}=$ desarrollo+econ\%C3\%B3mico\&hl=es\&sa=X\&ved=0ahUKEwiEwaK6kfHWA hXJKyYKHT4jBWQQ6AEIKjAB\#v=onepage\&q=desarrollo\%20econ\%C3\%B3 $\underline{\text { mico\&f }=\text { false }}$

\section{REFERENCES CONSULTED}

1. Aldana Zavala, J., \& Colina Ysea, F. (2019). Green marketing in the conformation of a planetary citizenship in the Latin American educational field. San Gregorio Magazine, 0 (31), 150-161. Recovered from http://revista.sangregorio.edu.ec/index.php/REVISTASANGREGORIO/article/v iew/972/15

2. Boza-Valle, J. A., \& Manjarez-Fuentes, N. (August 2016). Strategic diagnosis of enterprises of popular and solidarity economy in Ecuador. Scielo, XXXVII (2). Retrieved from http://scielo.sld.cu/scielo.php?pid=S1815$\underline{59362016000200010 \& s c r i p t=s c i}$ arttext\&tlng=en

3. ECLAC (2013). The economic development of Ecuador. ECLAC, Secretariat of the Economic Commission for Latin America and the Caribbean. Mexico: Editogran S.A. Retrieved from http://www.politicaeconomica.gob.ec/wpcontent/uploads/downloads/2014/02/librocepal.pdf

4. Coraggio, J. L. (1998). Urban economy: the popular perspective (Second ed.). (r. illustrated, Ed.) Quito, Pichincha, Ecuador: Editorial Abya Yala. Retrieved on April 20, 2016, from https://books.google.com.ec/books?id=UkE4pQZKColC\&printsec=frontcover\& 
$\mathrm{dq}=$ economia + popular $+\mathrm{y}+$ solidaria\&hl=en419\&sa=X\&ved=0ahUKEwiy0M6U25PLAhVBmh4th4QhqyqQ\&f======

5. Coraggio, J. L. (June 2014). The presence of the social and solidarity economy and its institutionalization in Latin America. ECONSTOR (7), 22. Retrieved from https://www.econstor.eu/bitstream/10419/148805/1/862974798.pdf

6. The Telegraph. (April 23, 2016). Manabí's economic wealth is focused on agriculture, fishing and tourism. News Writing. Obtained from http://www.eltelegrafo.com.ec/noticias/ecuador/3/la-riqueza-economica-demanabi-se-centra-en-la-agricultura-la-pesca-y-el-turismo

7. Guerra, P. (2012). The solidarity economy in Latin America. PAPERS, 76. Obtained from http://www.fuhem.es/revistapapeles/docprivados/110/la\%20economia\%20soli daria\%20en\%20Latinoamerica P.\%20GUERRA.pdf

8. Hernández Sampieri, R., Fernández Collado, C., \& Baptista, L. (2014). Investigation methodology. Mexico: Editorial Mc - Graw - Interamerican Hill. Sixth edition.

9. Kerlinger, F. \& Lee, H. (2008). Behavioral research: Research methods in Social Sciences (4th ed.). Mexico: Mc Graw Hill.

10.Organic Law of the Popular and Solidarity Economy. (April 14, 2011). 48. Quito, Pichincha, Ecuador. Retrieved from http://www.seps.gob.ec/documents/20181/25522/Ley\%200rga\%CC\%81nica \%20de\%20Economi\%CC\%81a\%20Popular\%20y\%20Solidaria.pdf/0836bc47bf63-4aa0- b945-b94479a84ca1

11. Organic Law of the Popular and Solidarity Economy. (2011). Retrieved from http://www.seps.gob.ec/documents/20181/25522/Ley\%20Orga\%CC\%81nica \%20de\%20Economi\%CC\%81a\%20Popular\%20y\%20Solidaria.pdf/0836bc47bf63-4aa0- b945-b94479a84ca1

12.Mondéjar J., J., Vargas V., M., \& Meseguer S., M. L. (2009). Measurement of regional economic development through synthetic activity indicators. Venezuelan Journal of Joint Analysis, XIV (2). Retrieved from http://www.redalyc.org/articulo.oa?id=36414215

13. Ocegueda, J. M. (2000). Growth and economic development - the current state of debate. Baja California, Mexico: UABC. Retrieved from https://books.google.com.ec/books?id=y5fGeXERg1UC\&printsec=frontcover\& 


\section{CIENCIAMATRIA}

Revista Interdisciplinaria de Humanidades, Educación, Ciencia y Tecnología

Año VI. Vol. VI. №10. Enero - Julio 2020

Hecho el depósito de ley: pp201602FA4721

ISSN-L: 2542-3029; ISSN: 2610-802X

Universidad Nacional Experimental Francisco de Miranda (UNEFM). Santa Ana de Coro. Venezuela

Diana Asunción Bravo Vélez; Carlos Artemidoro Zea Barahona; Jose Alfredo Blanco Yoza; Tatiana Lisbeth Mero Flores

$\mathrm{dq}=$ development+econ\%C3\%B3mico\&hl=en\&sa=X\&ved=0ahUKEwiks4ngqvb YAhUH1IMKHatHDulQ6AEIJTAA\%\%d\%3d\%3

14.Quevedo Vázquez, J., Vásquez Labefre, L., \& Quevedo Barros, M. (2019). Analysis of business incubators in Ecuador. Interdisciplinary Arbitrated Review Koinonía, 4 (8), 633-648. doi: http: //dx.doi.org/10.35381/r.k.v4i8.318

15.Superintendence of popular and solidarity economy (2019). Know what the Popular and Solidarity Economy (EPS) is. Recovered from https://www.seps.gob.ec/noticia?conoce-la-eps

16.Zermeño, F. (2004). Lessons of economic development. Plaza and Valdes. Retrieved from https://books.google.com.ec/books?id=Iv24eXOtNZ8C\&printsec=frontcover\&d $\mathrm{q}=$ development+econ\%C3\%B3mico\&hl=en\&sa=X\&ved=0ahUKEwiEwaK6kfH WAhXJKyYKHT4jBWQQvAdj\%20d3dEfjd=d3 